\title{
Internet of Things (loT) in Agriculture Industries
}

\author{
Erry Yulian Triblas Adesta ${ }^{1}$, Delvis Agusman ${ }^{2}$, Avicenna ${ }^{1}$ \\ ${ }^{1}$ Department of Manufacturing and Materials Engineering \\ Faculty of Engineering, International Islamic University Malaysia \\ Jalan Gombak 50728 Kuala Lumpur \\ ${ }^{2}$ Department of Mechanical Engineering \\ Faculty of Engineering, Universitas Muhammadiyah Prof. Dr. HAMKA, Jakarta \\ e-mail: eadesta@iium.edu.my
}

\begin{abstract}
The IOT applications in the agriculture industry are intertwined in many ways, creating a hybrid construct of traditional businesses with digital connectivity implementations. Strategic issues and principles on the assimilation of loT solutions are prevail through time: investment costs, data interconnectivity, management and analysis, as well as data security. The agriculture industry is expected to be always alerted for the changes that may apply, with all the contemporary challenges and future directions responding to the situations, so it can reap the optimum use of the loT connectivity.
\end{abstract}

Keywords: Internet of Things, Agriculture, Agro Industrial Systems

\section{Introduction}

\subsection{Internet of things and industrial Big Data}

A world where all objects the human capable of interacting are connected to the network, and generating and collecting data for a decision-making purpose is not far beyond mankind grasp. The early stage of such aspired world has already begun when the concept of Internet of Things (IOT) was introduced for the first time in 1999 where the phrase was originally started as the title of a presentation by Kevin Ashton [1] at Procter \& Gamble (P\&G). The definition by Alkhalil \& Ramadan [2] for what we call as the loT today may describe and generally fits what is needed in this study: 'A worldwide network of interconnected entities'. The core concept of the term describes itself as a global infrastructure for the information society, with enabled advanced services by interconnecting physical and virtual things based on existing and evolving interoperable information and communication technologies [3].

In the loT, 'things' are defined as objects of the physical world (physical things) or of the information world (virtual things) capable of being identified and integrated into the information and communication networks [4]. The characteristic of loT lies on the entities that are capable of transferring data across networks in the absence of human-to-computer interaction [2]. It is realized in environments with trillions of device and information objects are connected through networks. Thus, loT can also be understood as a networking infrastructure for cyber-physical systems (CPS) that can be identified as an engineered system that is built from the seamless integration of computational algorithms and physical constituents [5]. Fundamentally, it is the 'interconnectivity' and 'things-related service', where any object will have the potential to be interconnected to the communication infrastructure, and proficient of providing thing-related services within the constraints of the things [6]. The transition starts with more and more devices, manufacturing tools and equipment, plants as well as vehicles, to be equipped with sensors [7]. Supporting not only limited to individual scope but also companies, to start optimizing their performance by gathering and analyzing data through the whole product lifecycle.

With the advent of the fourth industrial revolution, all industrial systems will be transformed into digital ecosystems. Thus, the emergence of loT resulted in the generation of large volumes of data exploding at really high rates [8], where by 2020 is predicted to exceed 40 trillion gigabytes (or 40 yottabytes). The collection of these vast amounts of data is usually referred to as "big data". The basic attribute that makes Big Data generated from the loT unique are the high volume, velocity, and variety of information, with new characteristics have been continuously introduced with the value being the most important [9]. Today, more than $98 \%$ of 
global information is being stored in digital format. Experts predict that the amount of stored information will be 20,000 times larger in 2045 [10].

The relation of IOT and the industry is the main concern in this study. With all the benefits that can be taken in, an attempt is necessarily required to intermingle what today classified as digital business model patterns with those from the non-digital world to create a hybrid construct of business. The value of an loT solution in the industry is found in combining a classic type of industry that in the past was not linked to the internet but now is upgraded with information technology and connectivity [11].

Both loT and Big Data will pose a key role in the future. The steadily growing amount of data will enable mankind to predict the spread of diseases, foresee where natural disasters will strike, and in time it may even be possible to predict human behavior [10]. The loT will collect even more information and feed directly into Big Data concept, where to provide meaningful information from the abundant data requires a special manipulation and analysis [12]. Presently, the loT remains a wide-open playing field for enterprises. It is still in its early stage, heterogeneous, and full of uncertainty. It is estimated that the potential economic impact by 2020 range from about USD 2 trillion to more than USD 14 trillion [13].

\subsection{State-of-the-art agro-industrial system}

The matter of discussion in agriculture industry varies in a wide spectrum, and the agroindustrial systems are intertwined in many ways, from the trade-in raw materials to final products [10]. Issues are there and prevail through time; from farmers issue to establish local food systems with incomplete product-related data; who delivers to whom? How are products delivered? And what are the transport costs and other expenses? [14], food processing technologies and their acceptance at consumers, to another contrast issue of environmental sustainability, e.g., in the events of extremes drought and moisture, due to the impact of agricultural activities [15]. However, there is one affinity that relates one issue of a particular sector to another. It is that most of their efficiency, performance, and productivity can be improved by the integration of automation and information technology to overcome the distinctive issues that hinder the sector.

It is relatively easy to find the assimilation and adoption of IoT in the developed and big scale agricultural sectors. It is not the infancy phase that the agriculture industry has been developing from typically conventional practices towards the utilization of sensors and other technologies. The main industry itself has been starting its upgrade years back where at the later time some agricultural companies, like Deere \& Company, started to commercialize the advance technologies for agricultural, construction, and forestry machinery. It is expected that the rapid evolution of science and technology in agroindustry, along with the recent advances in computer sciences and information technologies forms the basic foundations for the adoption of Cyber-Physical Agro-Industrial System. From a mundane and small application in a greenhouse farming, such as intelligent environmental control system for precision culture [16], to a larger application in agricultural zone management to search specific locations that fit with plant growth condition and environmental conditions [17].

There has been an example of a study by Zhou et al. [18] on the readiness of major technologies of IoT to be employed into agricultural system. With an approach from the perspective of the technological aspect itself, technology system framework of loT should be capable of solving defects in the traditional monitoring system and make public capable of acquiring agricultural information at any time. The claimed integrated system was said to be capable of realizing the automatic configuration and self-organization transmission of information collected node, thus expressing and storing the obtained agricultural information.

This study aims to evaluate the transition and the implementation of loT in agriculture industry. Strategic issues on the adoption of the loT are discussed in Section 2 to review the critical points and principles when one agribusiness adopts the technology. Section 3 provides the contemporary challenges of the loT and predict how the future researches and policies will be directed to answer those challenges. 


\section{Strategic loT solutions for agriculture industry}

The internet of things (loT) has been transforming the agriculture industry, allowing farmers to cope with the enormous challenge they face. The methods for the implementation with the definite roadmap are circumstantial and general principles without distinctive details may provide visions that allow stakeholders to develop a better loT strategy customized to their needs. Embedding the technology and successfully implementing the strategy for increasing the quality, quantity, sustainability and cost effectiveness of agricultural production are the main goals for those who would like to start integrating the conventional system with the loT solutions.

\subsection{Embedding the loT into agriculture industry}

Agriculture industry applies sensors, monitors, and other devices to improve their own operation and optimization of business, without developing and implementing the underlying technology of digital information. Thus Burkitt [13] coined the term 'embedding' as the nature of their business does not involve the activity of providing or enhancing the loT so other users can benefit from their own system.

In 2016, Poppe [19] presented in the workshop of the Organisation for Economic Cooperation and Development (OECD) that the implementation of the loT can support the application of smart farming, agri-food supply chains, and consumer interaction. This leads to several business models the loT can be embedded into the agriculture industry. In the developed countries, for examples, John Deere leads with the product innovation through heavy investments in the machinery industry. While basic data sales in the utilization of commercial equivalent open data have been dominated by Farmobile company. Another type provided by Monsanto company with value chain integration (e.g., Monsanto's Fieldscript) who provides Integrated Farming Systems (IFS) research platform, supplying a variable rate planting prescription for farmers' individual fields.

Few examples of typical embedded loT technology into farming systems were presented by Romeo [20] on Sensing Technologies for Effective Land Management Workshop. These emphasized the low power wide area (LPWA) applications for farming such as water metering for remote monitoring of water meters and leak detection and precision livestock for tracking animals grazing in the open pastures.

In the research scale, a study by Liu [16] for the precise culture of grape (Vitis vinifera 'Wink') in a greenhouse was conducted with a design of an intelligent monitoring-environmental control system. Sensors were fully equipped and located to obtain multiple sources of data. The data were then fused at the gateway to be a universal type before they were stored, analyzed, and modified. Monitored factors included the dynamic of grape growth, air temperature, air humidity, soil moisture, illumination, and $\mathrm{CO} 2$ concentration.

The study on precision farming also has been known for embedding the loT technology into their system. The precision agriculture involves the evidence-based farm management coupled with records on cultivation that resulted in information-added produce and informationoriented fields. It involves a thinking process of (1) describing facts and evidence with spatiotemporal coordinates to comprehend variability in the field, (2) making decision and action at the correct time and location, (3) recording the results of executed actions, and (4) reviewing the approaches by field-base geographic information system (GIS) [21]. A Japanese agricultural corporation, Aguri Co., Ltd., has experienced a strategy of precision paddy management. They have been keeping quality and yields of production with the data collected using the real-time soil sensor from the fields with more than 5-year organic management. The strategy resulted in records of big size data of field-level GIS which is applicable to decision support of farmers.

\subsection{Principles for loT strategy implementation in agriculture industry}

Implementation and integration of loT should be reflected in the improvement of production and service, whereby good implementation of key technologies \& infrastructure, data management, and analysis, should resulted in the accurate decision making for enterprises. In order to successfully integrating loT solutions into the business thus optimizing the results, there are several principles should be addressed. Some contents of section 2.2.2 to 2.2.6 were adapted from Burkitt [13]. 


\subsubsection{Key technologies and infrastructure}

For the agricultural sectors to embrace transition from conventional practices to digitalization, progress in certain technologies contributes significantly to the IoT being implemented. The deployment of these IoT technologies will significantly impact and change the way enterprises do business, thus affecting many processes inside. To reap the many potential benefits that have been postulated, the initial processes of designing the architecture of the system, infrastructure and hardware installation, and finding the right sensors and instruments are critical to be taken into account. It also must be highlighted that sufficient infrastructure and communication technologies, starting from machine-to-machine (M2M) interfaces and electronic communication protocols, wireless communication, RFID technology, energy harvesting technologies, sensors and location technology, as well as software should be maintained in good condition [22].

\subsubsection{Addressing the industries and markets}

A thorough assessment by business process modeling should be done towards how the business environment is being transformed by the loT. This covers the assessment on how the services and products are expected to be combined, or affected by the loT. How far the demand of the company to transform the activities inside may be affected by the loT availability that already exists in the industry. It is suggested that the more the loT technology exists, the more rapidly the business should have to move [13].

\subsubsection{Business engagement}

It has to be highlighted that value in the loT will be created through the transformation of customer experience. With that reason, the establishment of loT technologies should be formerly initiated from a strong capability in experience design. It is important to assess the issue on what capabilities the company already had in this area, and what should be further developed. With a direct customer contact and when the opportunities for engagement appear, the loT could eventually transform the business.

\subsubsection{Connected products and services}

If products and services have been established prior to loT implementation in the business, assessment to how can the two are integrated and enhanced with loT connectivity should be performed. The new ones could be then developed expressly for the loT. When new launches and innovations involved, the establishment of the connectivity, the analysis of the resulting data, and which other companies should the business collaborate with, must be taken into account immediately.

\subsubsection{Enhanced connectivity}

When initial wave of basic connected devices and services have been employed, a business may consider building further services by using analytics to gain insights from the big data the loT provides them. For example, when a greenhouse of an agricultural company gains a periodical environmental data for the growth of exotic plants, they may offer analytics with a certain value, for other companies who have the same interest of growing a similar commodity. As the level of loT deployment unfolds in agriculture, companies will usually look for ways of increasing value. Sometimes, new business models may emerge as the result of such activity.

\subsubsection{The capabilities of the organization}

Organizations' capabilities become the most critical part of the quest improving the company through loT. In short, the company who would like to employ connectivity should be able to distinguish itself in this space, assessing on what other company does and does not in the same field. Technically, improvements and investments aspects should be detailed before the transition started. Time, money and attention should be defined on where they are coming from, and what activities should be divested and downplayed so the resources can be allocated appropriately [13].

The company should also learn to develop "table stakes" capabilities. These include the skill to manage and analyze a vast number of data, integrating diverse portfolios, and to build business relations with loT-related companies. Without these capabilities, the company will be burdened to hire third-party to execute all the tasks mentioned. 


\section{Challenges and future directions}

The connectivity brought hailed as one of the greatest breakthroughs in the history of industrial technology. However, this innovation comes with forewarnings and specific requirements, and taking a full comprehension of the current situation and challenges will help the industry to provide a better direction in the future.

\subsection{Internet of things and its contemporary challenges}

There are several challenges in the implementation of loT connectivity today. Some are critical from agriculture point-of-view. The others may be approached from the side of the technology itself, from sensors data collection, communication relay, information analysis, and service layers.

In agriculture industry, the use of sensors is imminent and perpetual. An enormous number of sensors are regularly used, and the cost for installation and maintenance is a major concern. The larger the scale of the initial deployment of sensors into the business, the higher the amount of investment required, which is rare for a small scale agricultural-based business. In this context, Chen [23] responded to the situation by emphasizing to the sensors maintenance cost, that the challenge is to produce a technology that requires minimal or even zero effort to deploy and maintain. However, despite the high level of loT adoption in the industry, cost-efficient and plug-and-play approaches that allow systems interoperability are still missing [7].

\subsection{Data Interconnectivity}

The multitude of data generated by sensors when the system is running (e.g., sensors data on the plantation, environmental data, and consumer data in the market) is higher as the number of devices employed increased. The big data require careful management to ensure a smooth connectivity not only between the devices, but also communication to the middleware and to the central storage. Sometimes interconnectivity can be an issue when there is a barrier in the area due to the demographic coverage. For the areas with poor connectivity, Ferretti [24] describe the main communication scheme by focusing on communication technologies, wireless solutions such as sensor networks, ad-hoc and D2D communications, and multi-homing techniques (for customer) should be massively employed to guarantee the interconnection.

\subsection{Big Data Management and Analytics}

One of the main challenges, when loT started to be implemented, is the difficulties in capturing, visualizing, and analyzing the mega-data created by the sensors and devices [2]. Riggins [25][29] described the big data with high volume, velocity, and variety of information assets, requires a new form of processing to enable enhanced decision making, process optimization, and insight discovery. Thus, one of the main challenges is the visualization of the results. When a farming business generates data from the sensors on the field, the new approaches for context-aware visualization should be followed in order to support the decisionmaking in the different levels [7]. The fact is many farms did not survive the transition to large IT-controlled enterprises, and were subsequently acquired by IT specialists [10].

Since for most of the time the agriculture industry is not prepared for the experts that could store, manage and perform the data analytics, this can be a new issue. However, it can be approached by using services from the third party. A supporting vision was given by Mourtzis [7], who stated that the main aspects of industrial big data are not found inside one company but in a network of interconnected companies where different data should be collected in a place to process them, and derive meaningful insights for the decision-making. However, an alternative way can be used if the business has the capability of hiring people with the right competencies. Since in the more advanced agricultural system the works on the fields are automated, this made these days farmers are data analysts rather than people in overalls getting dirty.

\subsection{Data Security}

Data security is probably the most critical things to be secured when a business started to employ sensors and devices to collect data. Sensor readings from the plant growth, environmental data, and even customer-engagement data can be very critical for the company. Not only without the data the analytics could not be properly conducted, or probably resulted in

IJEEI Vol. 5, No. 4, December 2017: $376-382$ 
inaccurate information. Also if the data is compromised, it will be beneficial for those who obtained it. Either by selling the data to the competitors or probably by committing an unethical crime act of data ransom.

\section{Future directions for loT in agriculture industry}

There are key enabling technologies and application domains in the agriculture industry that are likely to drive loT research in the near future. The next generation Internet of Things will be directed to overcome the contemporary challenges and bring the innovations for future implementations of connected world. With the new technology to come, agriculture industry is expected to support the changes, participating in directing the future research for the benefits of the industry.

For the realization of loT vision on a decent interconnectivity with economical and lowcost technology, wireless communications, and digital electronics, varieties of technologies have been emerged. With the rapid development in the decades, agricultural sensing and networking technologies have been more and more mature. It has been observed by Lee [26], that hardware technology evolved by time. In the early presence, RFID tags and some sensors may be built by smaller and cheaper MEMs technology. In between 2010 to 2015, we observed some secure and low-cost tags development along with multi-protocol and multi-standards readers. Beyond 2020, it is predicted that new low-cost materials and nanotechnology will improve the evolution of the hardware.

Cloud computing answers the challenges arise in data management, as the most recent paradigm which promises reliable services delivered through next generation data centers that are based on virtualized storage technologies [27]. Magnetic recording has been the storage industry standard for decades. However, new emerging technologies such as bit-patterned media recording (BPMR), Shingled Magnetic Recording (SMR), and non-volatile memories (NVM) are bringing new feasible points for faster and higher-density storage [28]. It can be expected that in the near future, high reliability, scalability and autonomy to provide ubiquitous access, dynamic resource discovery and composability needed for the next generation loT applications can be achieved.

Security aspect will still be a major concern wherever massive connectivity is deployed. There can be many ways the system could be attacked-from accessing customer personal information, disabling network availability, or pushing erroneous data into the network [27]. These complex problems have solutions through cryptographic methods which deserved more research before they can be widely accepted. Data encryption ensures confidentiality against outside attackers, and message authentication codes safeguard data integrity and authenticity.

Agricultural data securities and privacy issues should always be protected. Future development in connectivity should always be followed. Keeping the update in terms of hardware and software technology may not completely eliminate the threats, but will push the imminent danger below the threshold.

\section{Conclusion}

The agriculture industry has been assimilating and adopting the loT for quite some time, transforming the typically conventional practices towards the utilization of sensors and other technologies. For this, the methods with a definite roadmap and general principles of loT strategies are circumstantial, as it allows one business to embed the technology and successfully, increasing the quality, quantity, sustainability and cost effectiveness of the company. However, the industry should always keep an eye open and observe relentlessly the challenges that come with the technology. The connectivity of loT may have passed the stage of infancy, and is still developing with promising potential for the agriculture industry to reap. 
References

[1] K Ashton. That 'internet of things' thing. RFiD J. 2011.

[2] A Alkhalil, R. Ramadan. IoT Data Provenance Implementation Challenges. Procedia Comput. Sci., 2017.

[3] O Vermesan, P. Friess. Internet of things-from research and innovation to market deployment. River Publishers. 2014.

[4] GM Lee, N Crespi, JK. Choi, M Boussard. Internet of Things. in Telecommunication Services Evolution, Springer, Berlin, Heidelberg. 2013: 257-282.

[5] "Cyber-Physical Systems (CPS) (nsf14542)," National Science Foundation, 2014. [Online]. Available: https://www.nsf.gov/pubs/2014/nsf14542/nsf14542.htm. [Accessed: 04-Sep-2017].

[6] Overview of the Internet of Things. International Telecommunication Union. 2012.

[7] D Mourtzis, E Vlachou, N Milas. Industrial Big Data as a result of loT adoption in manufacturing. Procedia CIRP. 2016

[8] R Teti, K Jemielniak, G O'Donnell, D Dornfeld. Advanced monitoring of machining operations. CIRP Ann. - Manuf. Technol. 2010; 59(2): 717-739.

[9] A Gandomi, M Haider. Beyond the hype: Big data concepts, methods, and analytics. Int. J. Inf. Manage. 2015; 35(2): 137-144.

[10] S de Wilde. The Future of Technology in Agriculture. The Hague: Stichting Toekomstbeeld der Techniek. 2016.

[11] E Fleisch, M Weinberger, F Wortmann. Business Models and the Internet of Things (Extended Abstract). in Interoperability and Open-Source Solutions for the Internet of Things, Springer, Cham, 2015: 6-10.

[12] B Bilbao-Osorio, S Dutta, B Lanvin. Rewards and Risks of Big Data. 2014.

[13] F Burkitt. A strategist's guide to the Internet of Things. New York: PwC Strategy\& Inc. 2014.

[14] J Lutz, B Smetschka, N Grima. Farmer Cooperation as a Means for Creating Local Food SystemsPotentials and Challenges. Sustainability. 2017; 9(6): 925.

[15] E Wheaton, S Kulshreshtha. Environmental Sustainability of Agriculture Stressed by Changing Extremes of Drought and Excess Moisture: A Conceptual Review, Sustainability. 2017; 9(6): 970.

[16] J Liu. Design and Implementation of an Intelligent Environmental-Control System: Perception, Network, and Application with Fused Data Collected from Multiple Sensors in a Greenhouse at Jiangsu, China. Int. J. Distrib. Sens. Networks. 2016; 12(7): 5056460.

[17] H Kang, J Lee, B Hyochan, S Kang. A Design of loT Based Agricultural Zone Management System. in ITCS \& STA 2012. Springer, Dordrecht. 2012: 9-14.

[18] H Zhou, B Liu, P Dong. The Technology System Framework of the Internet of Things and Its Application Research in Agriculture. Comput. Comput. Technol. 2011.

[19] K Poppe. Big opportunities for big data in food and agriculture. 2016.

[20] S Romeo. Enabling smart farming through the Internet of Things. 2016.

[21] S Shibusawa. Implementation of precision farming in green agro-industry concept. in Green AgroIndustry Investment For Our Future. 2013.

[22] Disruptive Technologies Global Trends 2025. in The Internet of Things, SRI Consulting Business Intelligence. 2010.

[23] YK Chen. Challenges and opportunities of internet of things. in 17th Asia and South Pacific Design Automation Conference. 2012: 383-388.

[24] S Ferretti, G D'Angelo. Smart shires: The revenge of countrysides. in 2016 IEEE Symposium on Computers and Communication (ISCC). 2016: 756-759.

[25] F Riggins, S Wamba. Research directions on the adoption, usage, and impact of the internet of things through the use of big data analytics. Syst. Sci. (HICSS). 2015.

[26] I Lee, K Lee. The Internet of Things (IOT): Applications, investments, and challenges for enterprises. Bus. Horiz. 2015

[27] J Gubbi, R Buyya, S Marusic, M Palaniswami. Internet of Things (IoT): A vision, architectural elements, and future directions. Futur. Gener. Comput. 2013.

[28] Y Sun, H Song, A Jara, R Bie. Internet of things and big data analytics for smart and connected communities. IEEE Access. 2016.

[29] PSG Aruna Sri, Anusha M. Big Data-Survey. Indonesian Journal of Electrical Engineering and Informatics (IJEEI). 2016; 4(1): 74-80.

IJEEI Vol. 5, No. 4, December $2017: 376-382$ 